WORKING PAPER - NO. 2020-26

\title{
Does Social Distancing Matter?
}

Michael Greenstone and Vishan Nigam

MARCH 2020

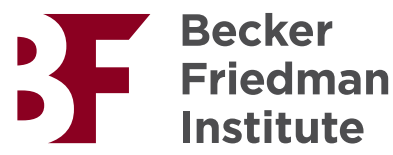




\title{
Does Social Distancing Matter? ${ }^{1}$
}

\author{
Michael Greenstone* \\ University of Chicago and NBER \\ Vishan Nigam \\ University of Chicago
}

March 2020

\begin{abstract}
This paper develops and implements a method to monetize the impact of moderate social distancing on deaths from COVID-19. Using the Ferguson et al. (2020) simulation model of COVID-19's spread and mortality impacts in the United States, we project that 3-4 months of moderate distancing beginning in late March 2020 would save 1.7 million lives by October 1 . Of the lives saved, 630,000 are due to avoided overwhelming of hospital intensive care units. Using the projected age-specific reductions in death and age-varying estimates of the United States Government's value of a statistical life, we find that the mortality benefits of social distancing are about $\$ 8$ trillion or $\$ 60,000$ per US household. Roughly $90 \%$ of the monetized benefits are projected to accrue to people age 50 or older. Overall, the analysis suggests that social distancing initiatives and policies in response to the COVID-19 epidemic have substantial economic benefits.
\end{abstract}

\footnotetext{
${ }^{1}$ Greenstone (mgreenst@uchicago.edu, corresponding author*) and Nigam (vnigam@uchicago.edu) contributed equally to this work. The authors declare no competing interests. We thank Claire Fan, Ian Pitman, Catherine Che, and especially Alice Schmitz for excellent research assistance; and Orley Ashenfelter, Magne Mogstad, Ishan Nath, Jonathan Cohen, Chinmay Lohani and Atakan Baltaci for several valuable conversations. All errors are our own.
}

Preprint: https://papers.ssrn.com/sol3/papers.cfm?abstract id=3561244

Code and data: https://www.michaelgreenstone.com/paperscategories\#vsl 


\section{Introduction}

The novel coronavirus (COVID-19) pandemic is considered the greatest public health threat since the 1918 Influenza Pandemic that infected one-third of the world's population and killed at least 50 million people. COVID-19 cases and fatalities are growing exponentially and there is much uncertainty about its ultimate impacts globally. Perhaps as unsettling as these estimates is the uncertainty around the health impacts that are wracking societies with fear.

In the absence of vaccines, countries around the world are implementing various forms of "social distancing" as a policy to slow the virus' spread. This social distancing takes many forms but, at its core, its aim is to keep people apart from each other by confining them to their homes in order to reduce contact rates. The impacts of social distancing are evident in the data from China, especially when compared with Italy which implemented social distancing policies more slowly and sporadically. At the same time, the economics costs are clear in the Chinese and Italian data, and in the US Goldman Sachs is projecting quarter on quarter annualized growth rates of $-6 \%$ in Q1 and -24\% in Q2 (Hatzius et al. 2020). Further, historically unprecedented US unemployment claims have begun to arrive and the near term outlook for the job market is grim (Hatzius et al. 2020). The demonstrated benefits in China (as well as South Korea and Singapore) and the sharp and large economic costs naturally raise critical questions about whether social distancing is worth it (Hilsenrath and Armour 2020; Bender and Ballhaus 2020; Thunstrom et al. 2020).

This paper develops and implements a method to estimate the economic benefits of social distancing. Our baseline finding is that a moderate form of social distancing is projected to reduce fatalities by 1.76 million in the next 6 months and that would produce economic benefits worth $\$ 7.9$ trillion. These benefits are over one-third of US GDP and larger than the entire annual federal budget. Distributed among US households, they are roughly equal to current median household income of $\$ 60,000$.

Further, these benefits are likely a lower bound. This is because they do not account for social distancing's impact on reducing uncertainty about mortality impacts, the potential for reducing morbidity rates, and improving quality of medical care for non-COVID-19 medical problems. It is also worth underscoring that the estimates depend on assumptions about the value of a statistical life (VSL) 
and estimated benefits remain substantial when other plausible assumptions are made. Finally, we find that the benefits from social distancing also remain substantial in less aggressive COVID-19 scenarios; for example, the benefits of social distancing are $\$ 3.6$ trillion even in a scenario where the peak of daily death rates is $60 \%$ lower than in the Imperial College model (Ferguson et al. 2020) of COVID-19 spread that we rely on in this paper.

The method has two main steps. First, we compare two scenarios from the prominent Ferguson et al. (2020) COVID-19 study: a mitigation scenario, which they define as "combining home isolation of suspect cases, home quarantine of those living in the same household as suspect cases, and social distancing of the elderly and others at most risk of severe disease" that lasts for 3-4 months, and a "no policy" scenario. The mitigation scenario is projected to reduce the number of COVID-19 caused fatalities by a total of 1.76 million over a 6 -month period, relative to the no policy scenario. This reduction in fatalities is composed of 1.13 million fewer deaths of COVID-19 patients treated in hospitals, particularly in intensive care units (ICUs); and 0.63 million fewer deaths of COVID-19 patients that are unable to receive ICU care because of pandemic-related overcrowding.

Second, the reduction in fatalities from the mitigation scenario is divided into 9 age categories and then monetized using the United States Government's VSL that we adjust for age (Thaler and Rosen 1976; Ashenfelter and Greenstone 2004; Murphy and Topel 2006; OMB 2003; US EPA 2015). In total, the benefits from the mitigation scenario equal $\$ 7.9$ trillion. Deaths avoided and monetized benefits are unequal: cohorts under age 50 comprise $11 \%$ of monetary benefits ( $3 \%$ of total deaths avoided); ages 50-69 comprise $52 \%$ of monetary benefits ( $28 \%$ of avoided deaths), and those 70 and older comprise $37 \%$ of monetary benefits ( $69 \%$ of avoided deaths). The differences in monetary benefits across age groups reflect that COVID-19 mortality rates are increasing in age while the VSL is generally decreasing in age.

Finally, we note that the particular benefits estimates are only as reliable as Ferguson et al.'s projections on COVID-19's spread and health risks. The method can be used with any set of projections, so as more information arrives and research advances, this approach can be applied to other projections and to infer the benefits of alternative policy responses.

The remainder of the paper is organized as follows. Section I describes our methods to project the direct 
and "overflow" COVID-19 caused deaths, based on Ferguson et al. (2020). Section II describes our approach to monetizing the avoided deaths in order to develop an estimate of the benefits of the mitigation social distancing scenario. Finally, Section III interprets the results, discusses some caveats, and concludes.

\section{Mortality Impacts of Social Distancing}

This section develops estimates of the projected mortality impacts of COVID-19, exclusively relying on Ferguson et al.'s (2020) "individual-based simulation model" that was developed to support pandemic influenza planning. The paper, which has been highly influential in the policy arena, combines data on early outbreaks of COVID-19 with demographic and hospital availability data from the United States to project the infection rates, hospitalization rates, demand for critical care, and mortality rates. It attempts to discipline these projections with data on COVID-19 experiences in China, Italy, Great Britain, and the United States (Ferguson et al. 2020).

Our emphasis is on Ferguson et al.'s "no policy" and mitigation social distancing scenarios. In the no policy scenario, there is uncontrolled growth of the coronavirus pandemic that leads to an $81 \%$ infection rate in the United States by October 1 and 2.2 million deaths. As a basis of comparison, in late February the CDC projected a $48-65 \%$ infection rate and deaths of 0.16 million (with a $0.25 \%$ infection fatality rate) to 1.7 million (1\% fatality rate) over a year starting March 2020 (Fink 2020). Importantly, other empirical studies now point to a case fatality rate close to $1 \%$ (Verity et al. 2020, Mizumoto and Chowell 2020), and other expert estimates suggest a 30-70\% US-wide infection rate (Axelrod 2020, Ramsey 2020). The Ferguson et al. estimates, while slightly more pessimistic, are thus broadly consistent with other projections of COVID-19 transmission.

The mitigation scenario emphasized by Ferguson et al. is a moderate form of social distancing that consists of 7-day isolation for anyone showing coronavirus symptoms, a 14-day voluntary quarantine for their entire household, and dramatically reduced social contact for those over 70 years of age. ${ }^{2}$ All measures begin in late March. The isolation and household quarantine measures are assumed to be in

\footnotetext{
${ }^{2}$ Ferguson et al. also model other subsets of mitigation, such as school and university closures, but these have limited impact and the mortality impacts are not emphasized.
} 
place for three months and reduced contact for people over 70 lasts four months. Ferguson et al. project that the mitigation scenario will reduce peak hospital demand by two-thirds and total deaths to 1.1 million.

We focus on the mitigation scenario because it approximates what the United States is implementing, albeit unevenly across the country. With perhaps the exception of California, Washington and New York, most US states have not pushed China-style shutdowns of the level necessary to suppress COVID transmission (Glanz et al. 2020). In other words, the US may "flatten the curve" of infection but not stop it entirely. Ferguson et al. also make projections about a "suppression" scenario that includes dramatically reduced contact for the entire population, and involves either a rebound epidemic (that strongly resembles our mitigation scenario) or repeated imposition of social distancing for two years. We view the latter as far from anything being implemented in the United States. One thing to note is that both the no policy and mitigation scenarios only extend through October 1 , so it is reasonable to assume that a vaccine will not be developed in this timeframe.

A novel feature of our analysis is that we improve upon Ferguson et al.'s estimated mortality projections by accounting for the potential shortages in the supply of hospital intensive care services, for example ICU beds, respirators, and trained staff. Specifically, Ferguson et al.'s headline death projections assume that all COVID-19 patients receive the appropriate medical care, so their projections do not account for potential shortages in ICU beds or respirators. Indeed, it is precisely the possibility of these shortages that account for the policy push to "flatten the curve" and avoid their repercussions. Our approach is to label the Ferguson et al. projections of deaths as "direct deaths" and develop projections of "overflow deaths" which are those that result from hospital ICUs reaching capacity and being unable to serve some COVID-19 patients. As we detail, we project that social distancing would reduce overflow deaths by an additional 630,000 fatalities.

In summary, we project that social distancing reduces COVID-19 caused deaths by 1.76 million deaths. This is composed of reductions of 1.13 million direct deaths and 630,000 overflow deaths. The remainder of this section describes how we develop these projections of the reductions in direct and overflow deaths due to social distancing and their distribution across 9 age categories. 
Fig 1. Modeling of Direct Deaths from COVID-19
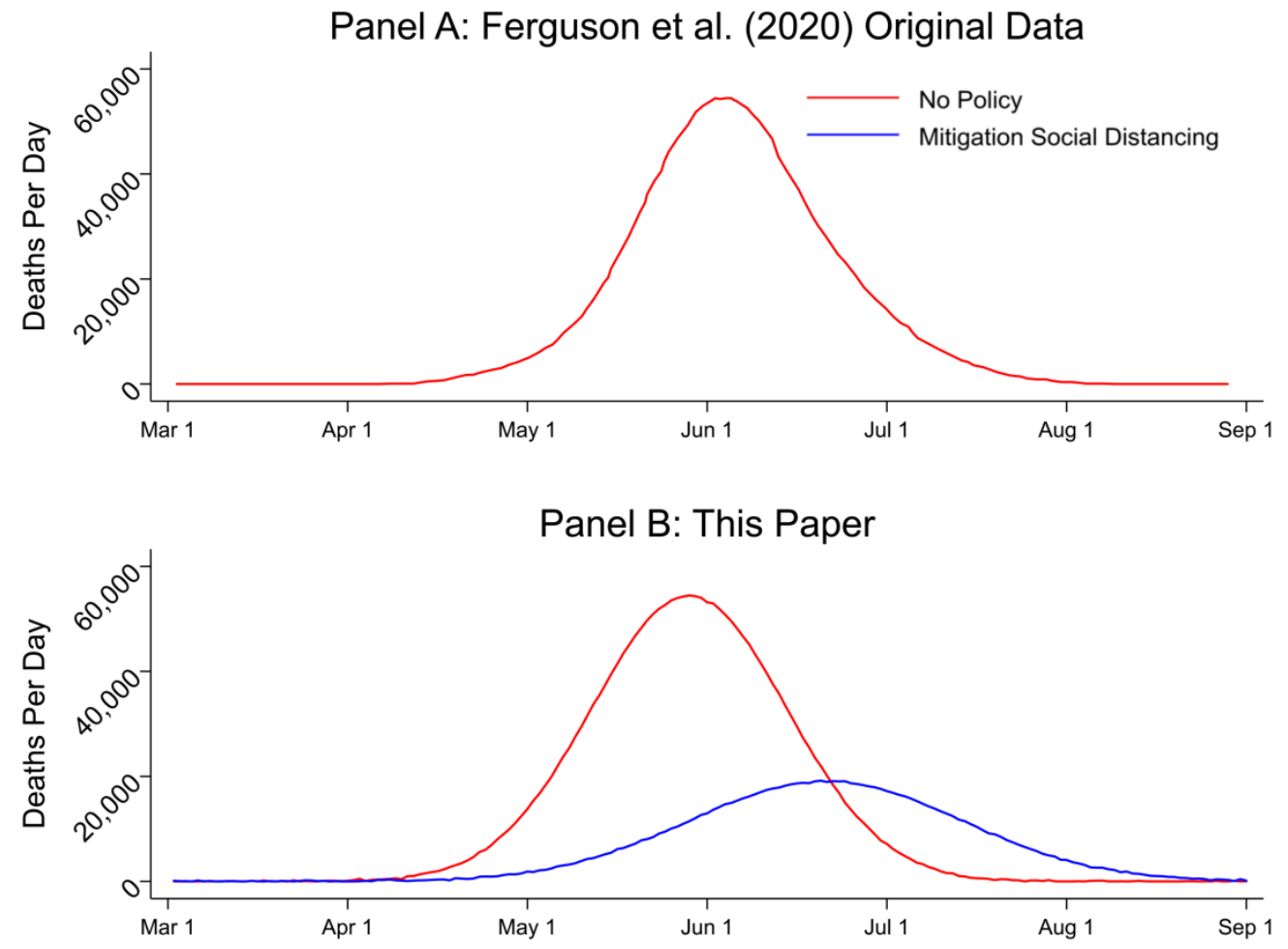

Notes: Figure shows how we construct daily direct deaths under various social distancing policies. The original distribution of US deaths with no policy from Ferguson et al. (2020) is given in Panel A. Panel B shows our normal approximation of this distribution, and a similar policy under mitigation social distancing. Total direct deaths (areas under the curves) are 2.2 million with no policy and 1.1 million with mitigation social distancing, exactly matching reported deaths in Ferguson et al.

\section{A. Direct Deaths}

We begin by reproducing the Ferguson et al. estimates of direct deaths in the US: 2.2 million with no policy and 1.1 million with "mitigation" social distancing. To do so, we develop a method that, under simple assumptions about the progression of coronavirus, allows us to construct the full daily distribution of deaths. This step is necessary because it was infeasible to acquire the full dataset underlying the Ferguson et al. analysis, undoubtedly due to the great demands placed on the authors as they model the progression of COVID-19 and replay updated findings to policymakers.

Our approach assumes that daily COVID-19 cases, deaths, and ICU bed demand follow a normal distribution. Normal distributions roughly approximate epidemic growth curves, which are slightly rightskewed since they grow exponentially until reaching herd immunity. Normality is also convenient because given the center (date of peak), height at peak, and width (distance from start to peak), it is possible to recover the full distribution (i.e., daily fatality counts). 
For an example of our strategy, consider Figure 1. Panel A reproduces Ferguson et al.'s distribution of daily deaths, which we extracted from their paper. ${ }^{3}$ The center of the distribution is around June 1 and the standard deviation visually appears to be about 16 days, so we can plot a normal distribution. Lastly, about 55,000 deaths per day happen at the peak. We then scale the entire distribution to peak at that value and sum deaths across all days to obtain total deaths from March 1 to October 1, 2020. So although we don't have the underlying data, we are able to reproduce this distribution with the red line in Panel B; our reconstructed version adds up to the same 2.2 million direct deaths that Ferguson et al. project for their no policy scenario by construction. ${ }^{4}$ The blue distribution in Panel B for daily deaths under the mitigation scenario is recovered with the same approach and, again by construction, produces exactly the 1.1 million deaths that Ferguson et al. project. ${ }^{5}$

The bottom line from this analysis is that social distancing is projected to reduce the number of COVID19 deaths by 1.1 million between March 1 and October 1 . This is simply the difference in the number of direct deaths in the no policy and mitigation scenarios.

\section{B. Overflow Deaths}

We next estimate ICU overflow deaths under the no policy and mitigation scenarios, as well as their difference which is the number of fatalities averted through COVID-related social distancing. We believe that these are the first projections of overflow deaths or, put more plainly, the mortality costs of failing to "flatten the curve". Previous work (e.g., Ferguson et al. 2020, Jha et al. 2020) project hospital bed and ventilator needs in excess of capacity, but do not project the impact of these shortages on total fatalities. ${ }^{6}$

\footnotetext{
${ }^{3}$ Panel A corresponds to the US curve in Figure 1a of Ferguson et al. (2020), which is expressed in deaths per 100,000 people; we multiply through by the US population to obtain total US deaths.

${ }^{4}$ For some distributions, we have even less information. The only Ferguson et al. (2020) plot showing curves with and without moderate distancing is for critical care cases in Great Britain, not deaths in the USA. However, that plot still lets us infer that the epidemic peak is one-third as high and takes $40 \%$ more time to occur relative to April 1 , and has a $40 \%$ larger standard deviation, compared to no policy. These points are sufficient to construct direct deaths with mitigation in the United States.

${ }^{5}$ We add a mean zero error to our reconstructed normal distributions, such as in Panel B.

${ }^{6}$ The challenge in estimating overflow deaths is that the death rate changes as a function of the number of patients, so a standard SIR model that takes COVID-19 death rates at an input will not directly capture this phenomenon. In contrast, empirical comparisons between overwhelmed and calmer hospital systems (ex: Wuhan vs. rest of China) are challenging because distancing policies are most severely implemented in overwhelmed areas, confounding comparisons.
} 
A little background on ICU services is helpful to understand this calculation. Patients in the ICU receive specialized beds, ventilators, and care from doctors and nurses with specialized training. The United States has 85,000 beds in intensive care units (Tsai et al. 2020). Of those, 32,000 (37\%) are unoccupied and immediately available to treat COVID-19 patients. The total number of beds that COVID-19 patients could fill, known as "surge" capacity, lies between the two. In times of emergency, some space can be made by canceling elective surgeries, but cancer patients and others with ongoing treatment must stay put. We follow Ferguson et al. in assuming ICU "surge" capacity of 45,000 beds ( $=32,000$ unoccupied ICU beds plus 13,000 ICU beds made available by canceling elective surgeries). ${ }^{7}$ Increasing ICU capacity any further requires new physical beds and equipment, as well as proportional increases in the number of ICU doctors and nurses.

The first step in projecting overflow deaths is then to project the number of ICU beds available each day for COVID-19 patients and the daily number of new patients in need of ICU care. We follow Ferguson et al. and assume that each ICU patient occupies a bed for exactly 10 days. Given the surge capacity of 45,000 ICU beds $^{8}$, this means that a total of 4,500 ICU beds become available each day for COVID-19 patients. Ferguson et al. projects the number of new COVID-19 patients that need ICU services each day for both scenarios. ${ }^{9}$

Figure 2 reports the results from this exercise. The dashed black line is the number of ICU beds that become available each day for COVID-19 patients in need of ICU-level care. The red and blue distributions are the number of new COVID-19 patients that require ICU services each day under the no policy and mitigation social distancing scenarios, respectively. The patients underneath the dashed black line receive ICU services, while those above it are projected to be denied them.

Under social distancing, 1.57 million fewer COVID-19 patients that merit ICU services are denied them. Specifically, the no policy number of COVID-19 patients in need of ICU services that are denied them is equal to the sum of the left ( 1.92 million) and center ( 0.60 million) shaded regions. In the mitigation social distancing scenario, this is equal to sum of the center ( 0.60 million) and right ( 0.35 million)

\footnotetext{
${ }^{7}$ The authors assume the US has 14 ICU beds per 100,000 people, which is 45,000 overall. This is slightly lower than the Tsai et al. (2020) estimate of 58,000 potentially available ICU beds.

${ }^{8}$ Ferguson et al. (2020) report that US ICU surge capacity is 14 beds per 100,000 people; we multiply by US population and divide by 10 days/ICU patient to obtain the ICU surge capacity shown in Figure 2.

${ }^{9}$ Plots in Ferguson et al. (2020) are of projected ICU beds occupied by day, which we divide by 10 days per ICU patient to obtain the number of new patients per day, as shown in Figure 2.
} 
Fig 2. Predicted ICU Patient Flows

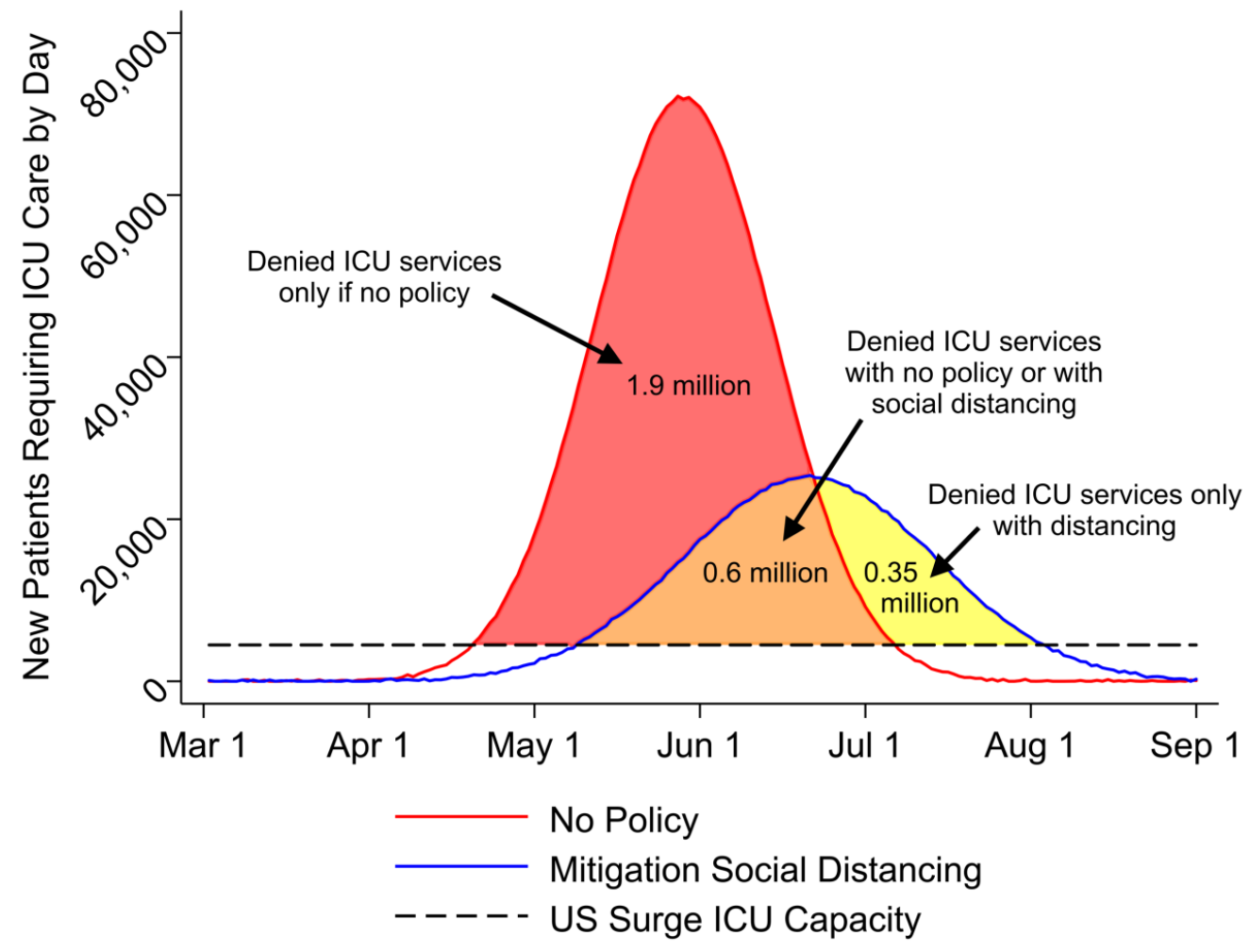

Notes: Figure illustrates our computation of overflow deaths from patients unable to receive ICU care. Daily flows of patients requiring ICU care are constructed from Ferguson et al. (2020) projections of bed demand. Patients above US surge capacity (black line) are denied ICU treatment: the red and orange areas with no policy and the orange and yellow areas with "mitigation" social distancing. The difference of 1.6 million represents COVID-19 patients denied ICU treatment, each of which has a $50 \%$ chance of survival with ICU treatment (Ferguson et al. 2020 ) and a $10 \%$ chance of survival of denied care.

regions. Therefore, the benefit of social distancing (i.e., the difference in these two numbers) is the difference between these two numbers or 1.57 million.

The prospects for these 1.57 million ICU indicated, but denied, patients are poor and we project that an additional 630,000 of them would die. This calculation requires an estimate of the difference in mortality rates for ICU-indicated COVID-19 patients who can and cannot get ICU services. We rely on Ferguson et al.'s assumption that the survival rate for ICU-level COVID-19 patients in ICUs is 50\% and our read of the literature that suggests that the survival rate falls to $10 \%$ or below if they are denied ICU services (Emanuel et al. 2020, Long et al. 2015). In summary, 1.57 million coronavirus ICU patients face a 
$40 \%$ higher death rate in the no policy scenario, relative to the mitigation social distancing scenario. Put another way, social distancing reduces the projected number of overflow deaths by 630,000 in the United States between March 1 and October 1, 2020, providing a quantitative rationale for efforts to "flatten the curve".

\section{Age Distribution of COVID-19 Deaths}

The next step in the analysis is to assign projected COVID-19 caused deaths - which we have computed for the entire US population - to age groups. Ferguson et al. (2020) report the distribution of total deaths from the no policy scenario across 9 age groups (i.e., $0-9,10-19, \ldots, 70-79$, and 80+). ${ }^{10}$ We apply this same distribution of total deaths to the mitigation scenario. This is not an innocuous assumption, because the marginal deaths in this scenario may have a different age distribution, but alternative information is unavailable. ${ }^{11}$

\section{The Monetary Value of Social Distancing}

This section describes our approach to monetizing reductions in fatalities and then uses it to develop an estimate of the benefits of the mitigation social distancing scenario, relative to the no policy scenario.

\section{A. The Value of a Statistical Life and the Monetary Benefits of Changes in Mortality Rates}

It is natural to consider social distancing like any of hundreds of policies that aim to reduce the risks that people face. As just one example of such policies, governments pay for guardrails on the side of roads, because they increase survival rates in car accidents. A policy like social distancing similarly increases survival rates.

To convert the main benefit of social distancing - reducing the mortality impact of COVID-19 - into dollar terms, we turn to the value of a statistical life (VSL). The VSL is a tool from economic theory which is now a standard ingredient in the cost-benefit analyses that undergird decision-making by the United States Government, and scores of foreign, state, and local governments (OMB 2003). In principle, the VSL measures how much the average US citizen is willing to pay for a reduction in the probability of

\footnotetext{
${ }^{10}$ Ferguson et al. report the infection fatality rate and probability of requiring ICU care by age group. We multiply each by 2017 age-group population from the US Census to obtain the age-wise distribution of direct and overflow deaths, respectively.

${ }^{11}$ In an extreme case, suppose distancing purely inhibits coronavirus from reaching nursing homes; if so, our approach will project deaths to elderly populations when in reality none have died.
} 
death. ${ }^{12}$ It is one statistical life, which is a reduction in mortality rates equivalent to saving one life on average. For instance, suppose the average American is willing to pay $\$ 10,000$ to avoid a $0.1 \%$ chance of death, then the VSL is equal to $\$ 10,000 / 0.001$ lives saved or $\$ 10$ million per statistical life saved. So, a policy that is expected to save 1 life has $\$ 10$ million in social benefits.

There are two reasons, one theoretical and one practical, to use the VSL to capture the benefits of social distancing policies. First, the VSL captures the full benefits an individual expects to derive from her own life, including from leisure, time with friends and family, and consumption of goods and services. The legal system often relies on individual's remaining lifetime earnings, but such a measure fails to capture many features of what people value about their life, including their consumption of non-market goods like leisure time spent with family members (Murphy and Topel 2006).

Second, our approach is a standard one: US federal agencies such as the EPA and Department of Transportation have used the VSL for many decades to evaluate a long list of policies in a variety of domains (transportation and environment are two common areas). These policies, like social distancing, have benefits measured in lower mortality but costs measured in dollars; the VSL allows the US government to compare the two, rather than neglecting that which cannot be valued.

In practice, we compute the social benefits of reducing COVID-19 mortality rates as:

$$
\text { Benefits }=V S L_{j} * \sum_{j}\left(R_{j}^{\text {direct }} * \operatorname{Pop}_{j}+R_{j}^{\text {overflow }} * P o p_{j}\right)
$$

where $\mathrm{j}$ is the age group. $R_{j}^{\text {direct }}$ is the reduction in the direct death rate from implementation of the moderate social distancing scenario, relative to the no policy scenario, that was outlined in Section I A. $R_{j}^{\text {overflow }}$ is the analog for ICU overflow deaths rate that was described in Section I B. Finally, Pop $p_{j}$ is the 2017 US population for the $\mathrm{j}$ age group and $V S L_{j}$ is the value of a statistical life that is allowed to vary with age. The VSL is allowed to vary with age following Murphy and Topel (2006), but we require the average for people 18 and over to equal \$11.5 million ${ }^{13}$ which matches the EPA's VSL for adults (US EPA 2015).

\footnotetext{
${ }^{12}$ It is important to underscore that the VSL is not the amount of money that a person would be willing to trade for certain loss of life (presumably all of their wealth) but rather for a small change in the probability of death. ${ }^{13}$ The US EPA employs a 2020 VSL of $\$ 9.9$ million in 2011 dollars as part of the Clean Power Plan Final Rule Regulatory Impact Analysis. This estimate accounts for income growth to 2020; adjusting for inflation, the VSL is $\$ 11.5$ million in 2020 dollars.
} 


\section{B. Empirical Estimates of the Monetary Benefits of Social Distancing}

Table 1 summarizes the paper's key results. The rows report on each of the 10 age categories and the US total. Column (1) reports the total US population. Columns (2d) details the projected reduction in direct deaths due to social distancing, with columns $(2 a)-(2 c)$ reporting the ingredients in this calculation. Columns $(3 a)-(3 d)$ repeat this exercise for overflow deaths. The total reduction in deaths due to social distancing (i.e., the sum of (2d) and (3d)) is reported in (4a). (4b) lists the age-specific VSL, which reflects the fact that income and remaining life expectancy vary across ages and many influence willingness-topay for reductions in mortality risk. To obtain column (4b) We obtain estimates of the VSL-age distribution from the authors of Murphy and Topel (2006) and rescale so that the population-weighted average for US adults (18+) equals the US EPA VSL of $\$ 11.5$ million. Finally, column (4c) reports the monetized value of the projected reduction in fatalities due to social distancing.

The topline result is that social distancing is projected to reduce COVID-19 caused fatalities by 1.76 million by October 1 and that this is worth $\$ 7.9$ trillion. This projected reduction in fatalities is composed of 1.13 million fewer deaths of COVID-19 patients receiving appropriate treatment (i.e., direct deaths) and 0.63 million fewer deaths of COVID-19 patients that are unable to receive ICU care because of pandemic related overcrowding (i.e., overflow deaths).

Figure 3 illustrates that the impacts are strikingly heterogeneous across age categories. People under the age of 50 have $\$ 0.85$ trillion (11\%) of total benefits, reflecting their low chance of death from COVID19. People aged 50-69 have $\$ 4.14$ trillion (52\%) of total benefits, almost double their share of deaths avoided through social distancing; in contrast, people 70 and older get $\$ 2.95$ trillion (37\%) of benefits despite comprising over two-thirds of deaths avoided. Cohorts aged 50-69 have larger total benefits than the 70+ group because the former have a higher VSL, reflecting the greater remaining life expectancies and expected future incomes of younger cohorts. More generally, it is apparent that COVID-19's risks and the benefits of social distancing are disproportionately concentrated among the older age groups. 
Table 1. Social Distancing's Projected Mortality Benefits and their Valuation in the United States

\begin{tabular}{|c|c|c|c|c|c|c|c|c|c|c|c|c|}
\hline \multirow[b]{3}{*}{$\begin{array}{c}\text { Age } \\
\text { group }\end{array}$} & Population & \multicolumn{4}{|c|}{ Direct Deaths } & \multicolumn{4}{|c|}{ Overflow Deaths } & \multicolumn{3}{|c|}{ All } \\
\hline & \multirow[b]{2}{*}{$\begin{array}{l}(1) \\
\text { US pop in } \\
\text { millions }\end{array}$} & \multirow{2}{*}{$\begin{array}{c}\text { No } \\
\text { Policy }\end{array}$} & \multirow{2}{*}{$\begin{array}{c}\begin{array}{c}\text { Mitigation } \\
\text { Distancing }\end{array} \\
(2 b) \\
\text { Pct } \\
\text { of pop }\end{array}$} & \multicolumn{2}{|c|}{ Difference } & \multirow{2}{*}{ 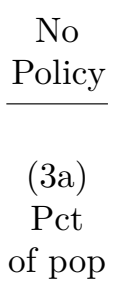 } & \multirow{2}{*}{$\begin{array}{c}\begin{array}{c}\text { Mitigation } \\
\text { Distancing }\end{array} \\
(3 b) \\
\text { Pct } \\
\text { of pop }\end{array}$} & \multicolumn{2}{|c|}{ Difference } & \multirow[b]{2}{*}{$\begin{array}{l}(4 \mathrm{a}) \\
\text { Death } \\
\text { count }\end{array}$} & \multirow[b]{2}{*}{$\begin{array}{c}(4 \mathrm{~b}) \\
\text { VSL in } \\
\text { million USD }\end{array}$} & \multirow[b]{2}{*}{$\begin{array}{l}(4 \mathrm{c}) \\
\text { Benefits in } \\
\text { trillion USD }\end{array}$} \\
\hline & & & & $\begin{array}{c}(2 \mathrm{c}) \\
\text { Pct } \\
\text { of pop }\end{array}$ & $\begin{array}{c}(2 \mathrm{~d}) \\
\text { Death } \\
\text { count }\end{array}$ & & & $\begin{array}{c}(3 \mathrm{c}) \\
\text { Pct } \\
\text { of pop }\end{array}$ & $\begin{array}{c}(3 \mathrm{~d}) \\
\text { Death } \\
\text { count }\end{array}$ & & & \\
\hline $0-9$ & 39.8 & 0.001 & 0.001 & 0.001 & 265 & 0.001 & 0.000 & 0.000 & 177 & 442 & 14.7 & 0.01 \\
\hline $10-19$ & 41.4 & 0.004 & 0.002 & 0.002 & 827 & 0.002 & 0.001 & 0.001 & 554 & 1,381 & 15.3 & 0.02 \\
\hline $20-29$ & 45.0 & 0.020 & 0.010 & 0.010 & 4,487 & 0.009 & 0.003 & 0.005 & 2,405 & 6,892 & 16.1 & 0.11 \\
\hline $30-39$ & 42.7 & 0.052 & 0.026 & 0.027 & 11,364 & 0.023 & 0.009 & 0.014 & 6,091 & 17,455 & 15.8 & 0.28 \\
\hline $40-49$ & 40.2 & 0.098 & 0.048 & 0.050 & 20,032 & 0.045 & 0.017 & 0.028 & 11,048 & 31,080 & 13.8 & 0.43 \\
\hline $50-59$ & 42.9 & 0.391 & 0.192 & 0.200 & 85,635 & 0.179 & 0.069 & 0.111 & 47,598 & 133,234 & 10.3 & 1.38 \\
\hline $60-69$ & 36.4 & 1.435 & 0.704 & 0.732 & 266,364 & 0.656 & 0.250 & 0.405 & 147,585 & 413,949 & 6.7 & 2.76 \\
\hline $70-79$ & 21.3 & 3.327 & 1.631 & 1.696 & 362,001 & 1.514 & 0.578 & 0.936 & 199,692 & 561,694 & 3.7 & 2.06 \\
\hline $80+$ & 12.4 & 6.067 & 2.974 & 3.093 & 382,484 & 2.791 & 1.066 & 1.725 & 213,339 & 595,824 & 1.5 & 0.89 \\
\hline US Total & & & & & $1,133,460$ & & & & 628,491 & $1,761,951$ & & 7.94 \\
\hline
\end{tabular}

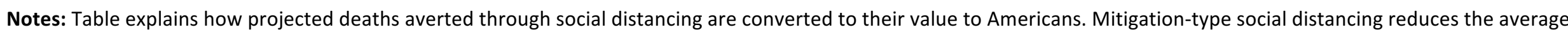

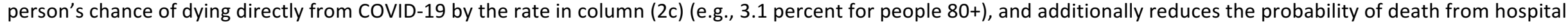

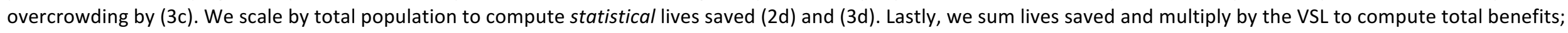

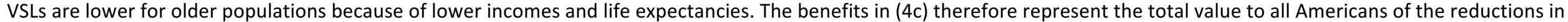
mortality risk in $(2 \mathrm{c})$ and $(2 \mathrm{~d})$, not the value of saving any particular life with certainty. 
Fig 3. Monetary Benefits of Projected Mortality Reductions from Social Distancing

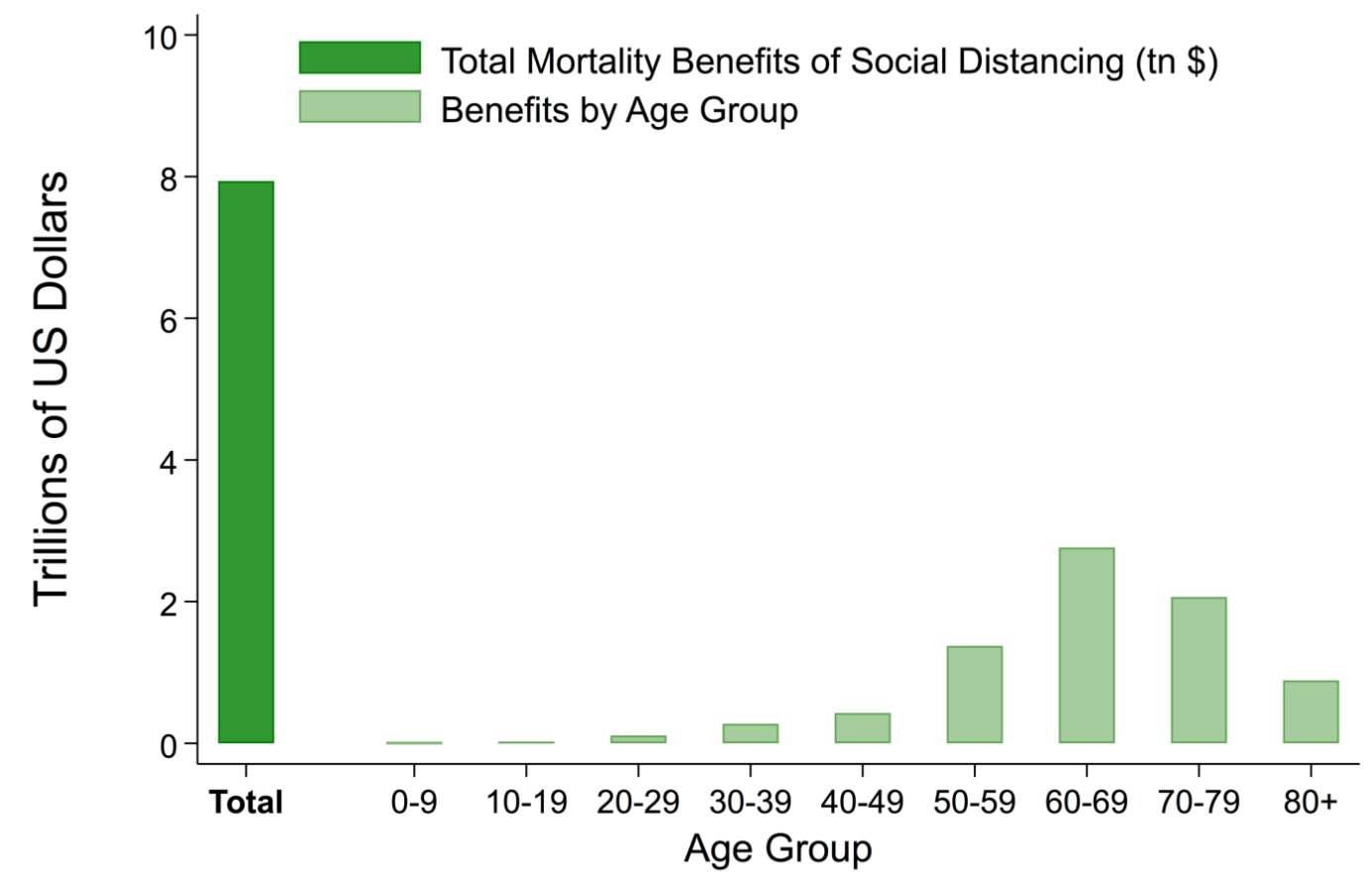

Notes: Figure shows total benefits (willingness-to-pay) for reduced COVID-19 mortality through social distancing. Total benefits of 7.94 trillion dollars equal the sum across age groups, where each age group's benefits are the change in expected mortality times the age-specific value of a statistical life. Despite facing lower mortality risk than above-70 cohorts, 50-59 and 60-69 year olds see large benefits because they have more years left to live and therefore higher VSLs.

\section{Robustness to Alternative Assumptions}

The credibility of the estimated $\$ 8$ trillion in benefits relies directly on parameters in the Imperial College model. This subsection examines how the monetized benefits of the mitigation social distancing scenario change under alternative assumptions about the virulence of the no policy scenario and surge ICU capacity, as well as the choice of an alternative VSL.

Table 2 reports on this exercise. Row (1) repeats the findings from this paper's baseline analysis. We consider what happens if the peak daily mortality rate is reduced, through any of a variety of mechanisms including lower infection rates and lower mortality rates conditional on infection. A reduction in the peak daily mortality rate by $30 \%$ reduces the benefits of social distancing to $\$ 6.5$ trillion (row (2a)), while a $60 \%$ reduction decreases it to $\$ 3.6$ trillion (row (2b)). Row (3) reveals that although doubling ICU capacity would meaningfully reduce the costs of COVID-19 it would have little impact on 
Table 2. Monetary Benefits of Projected Mortality Reductions from Social Distancing with Alternative Assumptions

\begin{tabular}{|c|c|c|c|c|}
\hline \multirow[b]{2}{*}{ Assumption } & \multicolumn{3}{|c|}{ Deaths Avoided Through Distancing } & \multirow{2}{*}{$\begin{array}{r}\text { Monetized Benefits } \\
\text { Benefits } \\
\text { (trillion USD) }\end{array}$} \\
\hline & $\begin{array}{r}\text { Direct Deaths } \\
\text { (millions) }\end{array}$ & $\begin{array}{r}\text { Overflow Deaths } \\
\text { (millions) }\end{array}$ & $\begin{array}{r}\text { Total } \\
\text { (millions) }\end{array}$ & \\
\hline (1) Main & 1.13 & 0.63 & 1.76 & 7.94 \\
\hline \multicolumn{5}{|l|}{ Lower Spread } \\
\hline (2a) Reduce peak of epidemic by $30 \%$ & 0.94 & 0.50 & 1.43 & 6.47 \\
\hline (2b) Reduce peak of epidemic by $60 \%$ & 0.57 & 0.22 & 0.79 & 3.56 \\
\hline \multicolumn{5}{|l|}{ More ICU Beds } \\
\hline (3) Increase Surge ICU Capacity by $100 \%$ & 1.13 & 0.63 & 1.77 & 7.97 \\
\hline \multicolumn{5}{|l|}{ Alternative VSL Assumptions } \\
\hline $\begin{array}{l}\text { (4a) Age-invariant VSL of } \$ 11.5 \mathrm{mn} \\
\text { (US EPA) for all deaths averted }\end{array}$ & 1.13 & 0.63 & 1.76 & 20.26 \\
\hline $\begin{array}{l}\text { (4b) Age-varying VSL with } \$ 3.5 \mathrm{mn} \\
\text { pop avg (Ashenfelter \& Greenstone 2004) }\end{array}$ & 1.13 & 0.63 & 1.76 & 2.42 \\
\hline $\begin{array}{l}\text { (4c) Age-invariant VSL of } \$ 3.5 \mathrm{mn} \\
\text { (Ashenfelter \& Greenstone } 2004 \text { ) }\end{array}$ & 1.13 & 0.63 & 1.76 & 6.20 \\
\hline
\end{tabular}

Notes: Table shows that that the total benefits of mortality reductions due to social distancing are similar under a series of alternative assumptions. (1) is the main estimate. In (2), we assume the peak of the epidemic, in terms of cases and deaths per day, was some fraction lower than in Ferguson et al. (2020). In (3) we double US surge ICU bed capacity and find a similar estimate of benefits, since ICU capacity increases lead to fewer deaths both with and without distancing. In (4a) we apply the US EPA 2020 VSL of \$11.5 million to all deaths averted, without accounting for patient age, and show that under US regulatory practice the estimated benefits would be over $\$ 20$ trillion. (4a) is analogous to (1) except that it uses a VSL of \$3.5 million, obtained from Ashenfelter and Greenstone (2004) and adjusted for inflation and income growth to 2020. (4c) is analogous to (4a) but uses the updated Ashenfelter and Greenstone (2004) VSL.

the benefits of social distancing. This may seem surprising, but it is because the benefits of additional ICU capacity are roughly equal in both the no policy and mitigation social distancing scenarios.

Lastly rows $(4 a)-(4 c)$, report the social benefits when alternative assumptions about the VSL are implemented. Row (4a) applies an age-invariant version of the US Government's VSL of \$11.5 million, rather than allowing it to vary with age as is done throughout the rest of the paper (US EPA 2015). In this case, the total social benefits are about $\$ 20$ trillion, more than 2.5 times larger than the baseline estimates. This finding is not surprising in light of the high proportion of saved lives that occur among people older than 60, who have relatively low VSLs in Table 1 because of their lower remaining life expectancy. While the age-invariant VSL has a legal basis in that it is US Government policy, it is challenging to justify from economic first principles of individual behavior. 
Rows (4b) and (4c) use an updated version of Ashenfelter and Greenstone's (2004) estimate of the VSL, which equals $\$ 3.5$ million when we adjust upwards ${ }^{14}$ for income growth to 2020 and convert into current dollars. This lower VSL naturally produces smaller estimates of the benefits of distancing. With age adjustment, the total social benefits are $\$ 2.4$ trillion, and without age adjustment they are $\$ 6.2$ trillion. It is evident that assumptions about the VSL play an important role in our exercise, but even at the lower end social distancing still produces benefits of several trillion dollars.

\section{Interpretation and Conclusions}

In this paper, we monetize one benefit of social distancing policies: a lower chance of dying from COVID19. Building on Ferguson et al., we show that a moderate social distancing scenario, implemented nationwide, is projected to save 1.76 million lives in the United States, including 0.63 million purely from shortages of hospital ICU beds. Applying estimates of the VSL based on economic theory and pegged to the US government VSL, the paper finds that Americans would be willing to pay approximately $\$ 8$ trillion for this reduction in mortality risk. Put another way, the estimated benefits of this mitigation social distancing scenario are roughly $\$ 8$ trillion.

It is worth taking a moment to contextualize this finding. \$8 trillion is over one-third of US GDP and larger than the entire annual federal budget. Put another way, the benefits of social distancing are roughly equal to current median household income of $\$ 60,000$. Whether in regular times or during a pandemic, it is difficult to think of any intervention with such large potential benefits to American citizens. Importantly, while we measure benefits of distancing in dollars, they reflect the high value Americans place on small reductions in their chance of death - including consumption, leisure, time with family, and other aspects of life not easily monetized.

It is likely that the $\$ 8$ trillion figure is an underestimate of social distancing's benefits because it misses several other channels. For example, the analysis does not account for the reduction in uncertainty around the mortality impacts of COVID-19, and valuing it in ways that reflect measured risk aversion would certainly increase the benefits. There is also the potential for social distancing to reduce the rates of non-fatal sickness experienced by the population, although this ultimately depends on the impacts on

\footnotetext{
${ }^{14}$ Consistent with existing literature (e.g., Carleton et al. 2019), we use an elasticity of the VSL with respect to income of unity to adjust the Ashenfelter and Greenstone (2020) VSL to the present.
} 
long run infection rates (Yang et al. 2020). Almond (2006) is an important data point, because it documents substantial long-run damages from in utero exposure to the 1918 influenza pandemic. Further, it seems reasonable to presume that social distancing will increase the quality of care for nonCOVID-19 medical problems by reducing the strain on medical providers, facilities, and supplies. Finally, it seems plausible that the changes in mortality rates being considered here are "non-marginal"; the available evidence suggests that the VSL is increasing for non-marginal changes in fatality risk, meaning that the analysis should use a larger VSL (Greenberg et al. 2020).

While it is tempting to undertake a full cost-benefit analysis of social distancing, this would require reliable estimates of its substantial costs. We are unaware of comprehensive estimates of these costs and their development is beyond the scope of our analysis, so the paper cannot go further than developing an estimate of the gross benefits of social distancing.

Finally, we are undoubtedly in the early days of learning about COVID-19 and the potential policy and societal responses. This paper's broadest finding is that it has developed a method to estimate the monetary benefits of social distancing and other policy responses to COVID-19 as they emerge. 


\section{References}

1. Almond, D. 2006. Is the 1918 Influenza Pandemic Over? Long-Term Effects of In Utero Influenza Exposure in the Post-1940 U.S. Population. Journal of Political Economy, 114(4), 672712.

2. Ashenfelter, O. and Greenstone, M., 2004. Using Mandated Speed Limits to Measure the Value of a Statistical Life. Journal of Political Economy, 112(S1), S226-S267.

3. Axelrod, Jim. March 2, 2020. "Coronavirus may infect up to $70 \%$ of world's population, expert warns," CBS News.

4. Axelrod, Carleton, T., Delgado, M., Greenstone, M., Houser, T., Hsiang, S., Hultgren, A., Jina, A., Kopp, R.E., McCusker, K., Nath, I. and Rising, J., 2018. Valuing the global mortality consequences of climate change accounting for adaptation costs and benefits.

5. Ferguson, Neil M., et al. March 16, 2020. "Impact of non-pharmaceutical interventions (NPIs) to reduce COVID-19 mortality and healthcare demand." London: Imperial College COVID19 Response Team.

6. Fink, Sheri. March 13, 2020. "Worst-Case Estimates for U.S. Coronavirus Deaths," The New York Times.

7. Glanz, James. March 20, 2020. "Coronavirus Could Overwhelm US Without Urgent Action, Estimates Say," The New York Times.

8. Greenberg, Kyle, Michael Greenstone, Stephen P. Ryan, and Michael Yankovich. 2020. "The Value of Statistical Life: Evidence from Military Retention Incentives and Occupation-Specific Mortality." Mimeograph.

9. Hatzius, Jan, et al. March 20, 2020. "US Daily: A Sudden Stop for the US Economy," Goldman Sachs.

10. Hilsenrath, Jon, and Armour, Stephanie. March 23, 2020. "As Economic Toll Mounts, Nation Ponders Trade-Offs," The Wall Street Journal.

11. Mizumoto, Kenji, and Chowell, Gerardo. 2020. "Estimating Risk for Death from 2019 Novel Coronavirus Disease, China, January-February 2020," Emerging infectious diseases, 26(6).

12. Murphy, K. M., and Topel, R. H. 2006. "The value of health and longevity," Journal of Political Economy, 114(5), 871-904.

13. Office of Management and Budget. 2003. "Circular A-4."

14. Pancevski, Bojan. March 22, 2020. "Covid-19 Deaths in Germany Far Below Rates in Other Countries," The Wall Street Journal.

15. Ramsey, Lydia. March 6, 2020. "One slide in a leaked presentation for US hospitals reveals that 
they're preparing for millions of hospitalizations as the outbreak unfolds," Business Insider.

16. Sanger-Katz, Margot, Kliff, Sarah, and Parlapiano, Alicia. March 17, 2020. "These Places Could Run Out of Hospital Beds as Coronavirus Spreads," The New York Times.

17. Thaler, R. and Rosen, S., 1976. The value of saving a life: evidence from the labor market. In Household Production and Consumption (pp. 265-302). NBER.

18. Tsai, Thomas, Jacobson, Benjamin, and Jha, Ashish. March 17, 2020. "American Hospital Capacity and Projected Need for COVID-19 Patient Care," Health Affairs.

19. U.S. Centers for Disease Control and Prevention. March 18, 2020. "Severe Outcomes Among Patients with Coronavirus Disease 2019 (COVID-19)-United States, February 12-March 16, 2020," Morbidity and Mortality Weekly Report, 69.

20. U.S. Environmental Protection Agency. 2015. "Regulatory Impact Analysis for the Clean Power Plan Final Rule."

21. Thunstrom, Linda, Newbold, Stephen C., Finnoff, David, Ashworth, Madison, and Shogren, Jason F. 2020. "The Benefits and Costs of Flattening the Curve for COVID-19."

22. Verity, Robert, et al. 2020. "Estimates of the severity of COVID-19 disease," medRxiv.

23. Viscusi, W. K., and Aldy, J. E. 2003. "The value of a statistical life: a critical review of market estimates throughout the world," Journal of risk and uncertainty, 27(1), 5-76.

24. Yang, Xiaobo, et al. 2020. "Clinical course and outcomes of critically ill patients with SARSCoV-2 pneumonia in Wuhan, China: a single-centered, retrospective, observational study," The Lancet Respiratory Medicine. 